\title{
Original
}

\section{Three-Dimensional Analysis of Pulp Chambers in Mandibular Second Deciduous Molars}

\author{
Yasutoyo Ikari ${ }^{1)}$, Hideaki Kinoshita ${ }^{1)}$, Hiroko Agematsu' ${ }^{1)}$, Hideki Saka ${ }^{2)}$, Satoru Matsunaga ${ }^{1)}$, \\ Yoshinobu Ide ${ }^{1)}$ and Shinichi Abe ${ }^{1)}$
}

\author{
1) Department of Anatomy, Tokyo Dental College, Tokyo, Japan \\ 2) Division of Forensic Odontology, Department of Dignostic \& Therapeutic Science, Meikai University School of Dentistry, Sakado, \\ Japan \\ (Accepted for publication, February 10, 2014)
}

\begin{abstract}
The aim of the present study was to clarify the shape of the pulp chamber and the three-dimensional positional relationship between the contour of the crown and pulp chamber using a micro-computed tomography system (micro-CT) for restorations of mandibular second deciduous molars without pulp exposure, and to evaluate quantitatively the thickness of the hard tissue surrounding each pulp chamber. Samples were cariesfree mandibular second deciduous molars of the deciduous and mixed dentition periods (10 each) extracted from the dry skulls of Indian children. Micro-CT images of the crowns were taken. Horizontal two-dimensional slice data were then stacked to construct three-dimensional images, and the shape of the pulp chamber and positional relationship between the contour of the crown and pulp chamber were observed from various directions. Then, the volume ratio of the pulp chamber relative to the crown contour and the distance from the crown surface of each pulp horn (hard tissue thickness) were investigated to evaluate the characteristics of the deciduous and mixed dentition periods objectively. The results showed that the mesiobuccal and mesiolingual pulp horns of deciduous dentition were most pointed, and approximately the same height. The pulp horn was lower and rounder in the mixed dentition period than in the deciduous dentition period. The pulp chamber shifted to the mesial side of the crown contour in both the deciduous and mixed dentition periods. These findings suggest that care should be exercised to avoid exposure of the mesial pulp horn during cavity preparation for deciduous mandibular second molars, especially during the deciduous dentition period.
\end{abstract}

Key words: Crown contour, Mandibular second deciduous molar, Three-dimensional observation, Pulp chamber, Micro-CT

\section{Introduction}

It has been reported that, in comparison with permanent teeth, deciduous teeth have pulp that takes up a larger volume of the crown, and have thinner enamel. ${ }^{1)}$ During cavity preparation for a deciduous coronal restoration, caution is needed to avoid pulp exposure. For this purpose, it is important to understand the morphological characteristics of a deciduous crown, create a stereoscopic image of the inner pulp chamber shape and pulp horn location from the crown surface under direct vision, and to know the tooth substance thickness in the pulp horn area.

The thickness of the tooth substance of deciduous teeth has been reported, but the studies concerned only took into account the thickness of the enamel or dentin. ${ }^{2,3)}$ Although a number of studies have measured hard tissue thickness in the pulp horn area

Correspondence to: Dr. Hideaki Kinoshita, Department of Anatomy, Tokyo Dental College, 2-9-18 Misaki-cho, Chiyodaku, Tokyo, 101-0061 Japan; Tel: +81 36380 9592; Fax: +81 3 6380 9664; E-mail: kinoshitahideaki@tdc.ac.jp of permanent teeth, ${ }^{4-7)}$ few have focused on deciduous teeth. ${ }^{8)}$

Some studies have also examined the positional relationship of the contour of the deciduous crown and the pulp chamber. ${ }^{9-11}$ ) However, the main methods employed were X-ray imaging and the use of sliced specimens, making it difficult to understand the images stereoscopically. Although one study employed threedimensional observation and morphological measurement, the approach involved preparation and optical superimposition of serial sections. This made it difficult to obtain accurate solid construction images and measurement data, as part of the sample was destroyed or lost during section preparation. High-resolution micro-CT allowing non-invasive stereoscopic observation from arbitrary directions has been used for three-dimensional observations of the deciduous crown contour and inner pulp chamber shape. ${ }^{12-14)}$ The present study focused on the shape of the pulp chamber, especially the pulp horn, and the positional relationship of the pulp chamber relative to the crown contour in mandibular second deciduous molars suggested that caries risk 


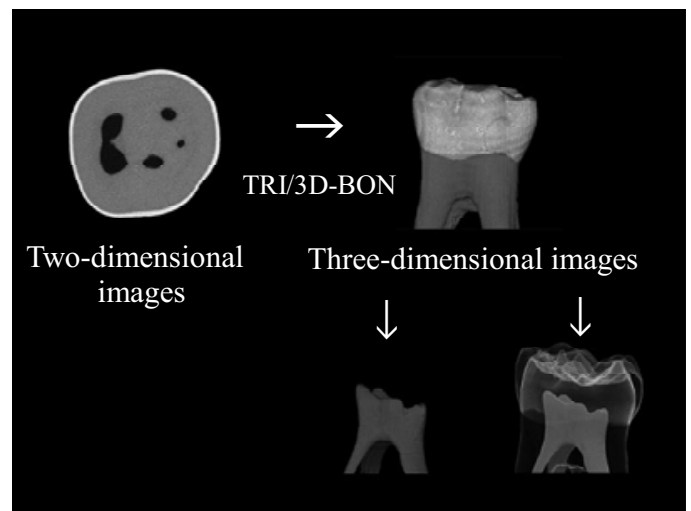

Figure 1. Procedure for constructing the three-dimensional images Three-dimensional images was performed using tow-dimensional images processed TRI/3D-BON software.

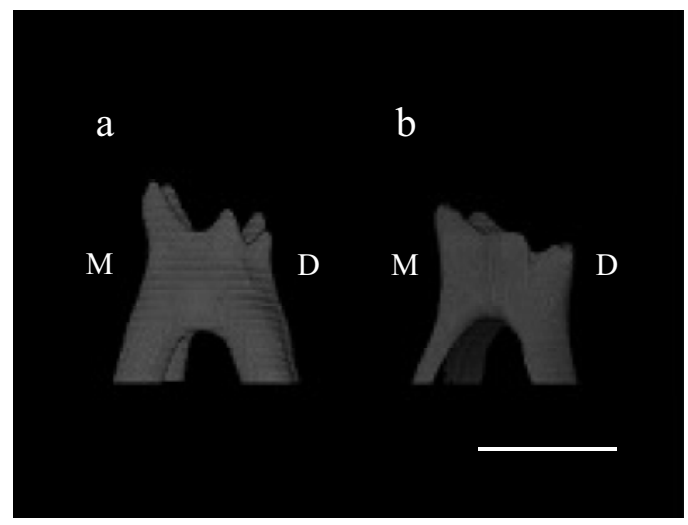

Figure 3. 3D rendered image of pulp chamber morphology of mandibular second deciduous molar

a: In the deciduous dentition, the pulp horns were projection.

b: In the mixed dentition, the pulp horns were rounded.

(Scale bar represents $5000 \mu \mathrm{m}$ )

was high during the deciduous and mixed dentition periods. Morphological measurements were also performed for objective evaluation. ${ }^{15}$

\section{Materials and Methods}

Twenty macroscopically caries-free mandibular second deciduous molars extracted from the dried skulls of Indian children were examined. Ten of the teeth were in the deciduous dentition period (when the central deciduous incisor to the second deciduous molar have reached the occlusal plane) and ten were in the mixed dentition period (when the central incisor, lateral deciduous incisor, deciduous canine, first deciduous molar, and second deciduous molar have reached the occlusal plane).

These teeth were scanned using a micro-CT system (HMX225 Actis 4, TESCO, Tokyo, Japan) consisting of an imaging device and a computer. The imaging device consists of an X-ray generator, a $360^{\circ}$ rotating specimen stage, and an X-ray detector. During imaging, the tooth specimen was placed with the tooth

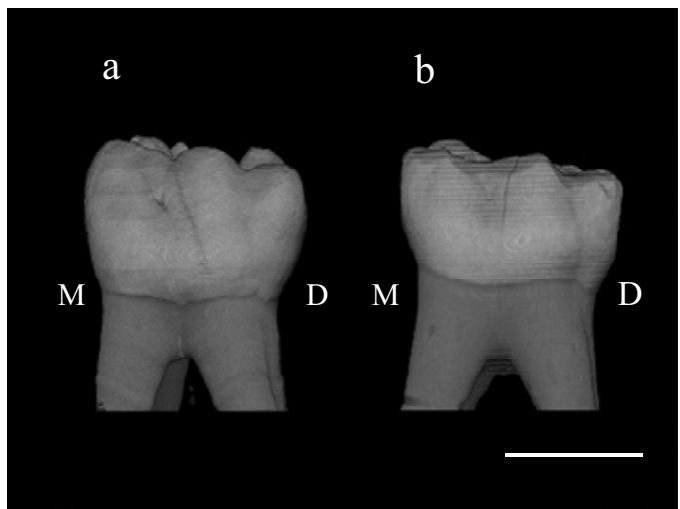

Figure 2. 3D rendered image of external crown morphology of mandibular second deciduous molar

a: In the deciduous dentition, apices of the cusps were projection. b: In the mixed dentition, apices of the cusps were attrition. (Scale bar represents $5000 \mu \mathrm{m}$ )

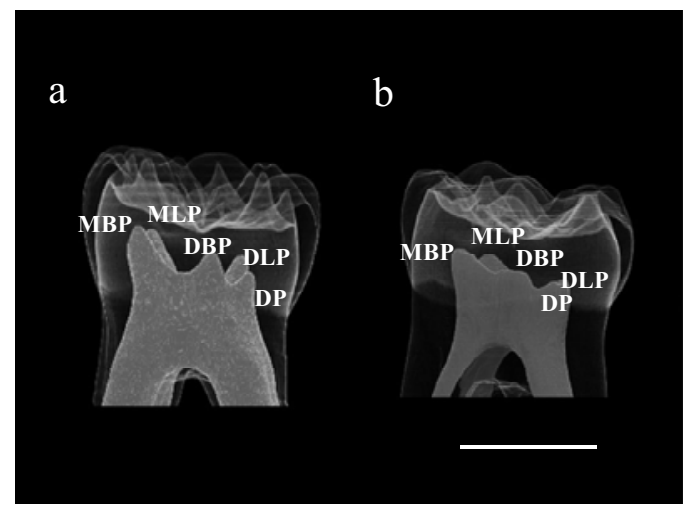

Figure 4. 3D topographic relationship of the crown contour and pulp chamber from buccal aspect

a: In the deciduous dentition, mesiobuccal and mesiolingual pulp horn were the highest.

$\mathrm{b}$ : In the mixed dentition, mesiobuccal and mesiolingual pulp horn were rounded.

(Scale bar represents $5000 \mu \mathrm{m})$

axis vertical to the platform. Imaging conditions were: tube voltage, $140 \mathrm{KV}$; tube current, $105 \mu \mathrm{A}$; focal spot size of the $\mathrm{X}$ ray generator, $5 \mu \mathrm{m}$; magnification, $\times 10$; and slice thickness, 50 $\mu \mathrm{m}$. The detector was equipped with an image intensifier tube measuring 4 inches and a 1-inch CCD camera with a scanning line of $1,024 \times 1,024$. The camera generated raw data comprising two-dimensional images.

Three-dimensional reconstruction was performed using twodimensional images processed using TRI/3D-BON software (RATOC, Tokyo, Japan). After different colors had been applied to the pulp chamber, dentin, and enamel in the three-dimensional reconstructed images to facilitate identification of each part, only the pulp chambers were sampled and observed from various directions. The enamel and dentin were rendered transparent and the topographic relationship between the contour of the crown and pulp chamber was observed from various aspects (Fig. 1).

In order to objectively evaluate the topographic relationship 
Yasutoyo Ikari et al.: 3D Analysis of Pulp Chamber in Deciduous Molars

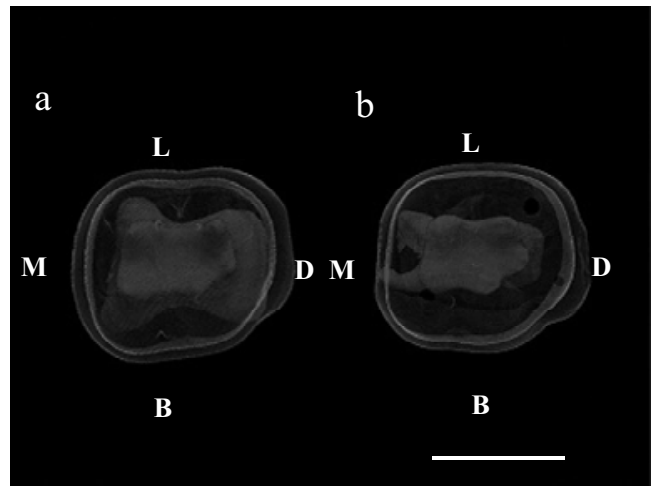

Figure 5. 3D topographic relationship of the crown contour and pulp chamber from occlusal aspect

a: In the deciduous dentition, the pulp chamber was shifted to the mesial side of the crown contour.

$\mathrm{b}$ : In the mixed dentition, the pulp chamber was smaller than deciduous dentition.

(Scale bar represents $5000 \mu \mathrm{m}$ )

$$
\text { [mm] } \approx: P<0.01
$$

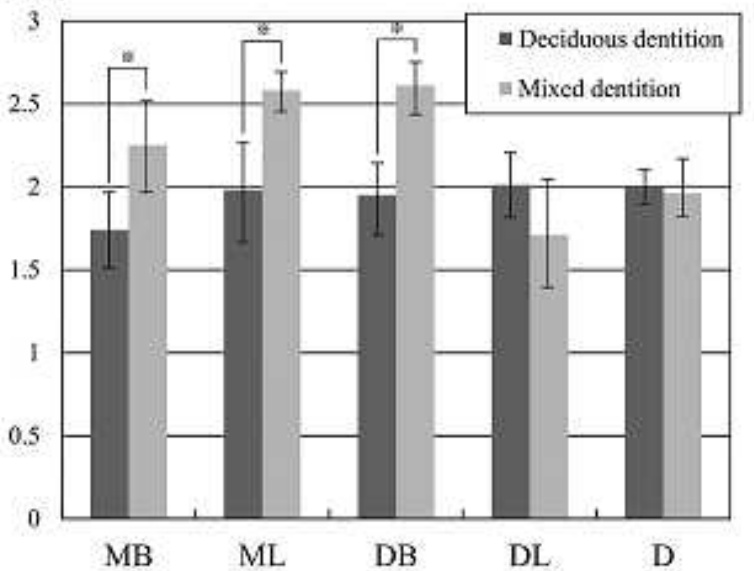

Figure 7. The dentin thickness at each of the pulp horns in the deciduous dentition and mixed dentition.

The dentin thickness in the cusp was the thinnest at mesiobuccal (MB) pulp horn in the deciduous dentition.

between the crown contour and pulp chamber, the volumes of the total crown and pulp chamber were calculated using the TRI/3DBON software. The volume ratio of the pulp chamber to the total crown was thus obtained. Furthermore, to clarify the thickness of the hard tissue at the pulp horn, arbitrary planes running through the pulp horns running parallel to the tooth axis were drawn in the reconstructed three-dimensional images, and morphological analysis was conducted to measure the distance between each pulp horn and the dentin surface. This research is allowed by our Institution.

The significance of differences in the results was examined in two dentition groups using the $t$-test at a significance level of $\mathrm{p}<0.01$.

\section{Results}

Crown contours of the mandibular second deciduous molars

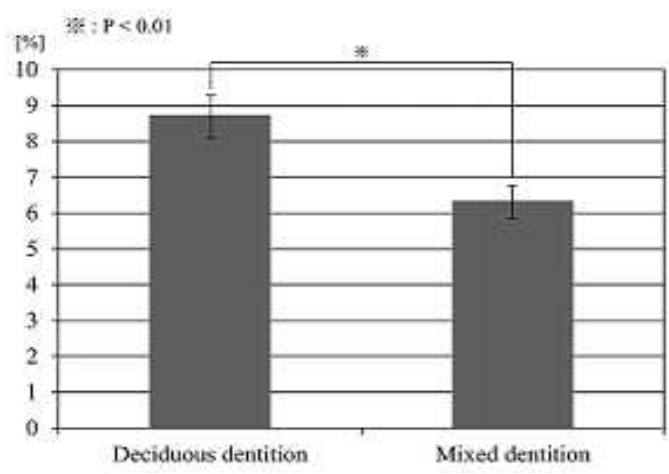

Figure 6 . The volume ratio of the pulp chamber to the total crown. The result were $8.8 \%$ in the deciduous dentition, and $6.3 \%$ in the mixed dentition.

in the deciduous and mixed dentition were observed from various directions. The mesiobuccal, distobuccal, mesiolingual, distolingual, and distal cusps were observed in both dentition periods. In the mixed dentition period, apices of the cusps were more rounded than in the deciduous dentition period (Fig. 2).

An image extracting only the pulp chamber revealed the shape of the 5 pulp horns (mesiobuccal, distobuccal, mesiolingual, distolingual, and distal pulp horns) in both the deciduous and mixed dentition periods. The pulp horns were clearly observed in the deciduous dentition period. The mesiobuccal pulp horn was highest, followed by the mesiolingual pulp horn. Among the three distal pulp horns, the distobuccal pulp horn was the highest, followed in order by the distal lingual and distal pulp horns. In addition, the mesiobuccal pulp horn showing an eminence on its mesial wall was observed in the deciduous dentition. In contrast, a significant change was observed in the internal pulp chamber compared to the crown contour in the mixed dentition period. The pulp horn became markedly lower in height and rounded. Such a change was especially noticeable in the three distal pulp horns (distobuccal, distolingual, and distal pulp horns) (Fig. 3 a, b). The shape of the distal pulp horn, which was smallest and lowest during the deciduous dentition period, was barely recognizable. These changes in the pulp horns were more significant than those in the crown contour.

These results indicated that the mesiobuccal and lingual pulp horns were the highest and intruded (Fig. 4 a, b) in the deciduous dentition period. In the mixed dentition period, the percentage of the pulp chamber relative to the crown contour became smaller. When observed from the occlusal surface in the deciduous dentition period, the pulp chamber was shifted to the mesial side of the crown contour (Fig. 5 a, b).

The proportional volume of the pulp chamber relative to the crown volume was measured for comparison to objectively evaluate the morphological findings for the mandibular second deciduous molar pulp chamber in the deciduous and mixed dentition periods. The values obtained were $8.8 \%$ in the deciduous 
dentition period, and $6.3 \%$ in the mixed dentition period, suggesting that the proportion of the pulp chamber volume was significantly higher during the deciduous dentition period (Fig. $6)$.

In addition, the thickness of the dentin in each pulp horn area was measured to investigate the change in thickness of the dentin (tooth substance) of each pulp horn area in the deciduous and mixed dentition periods. The corresponding values were $1.7-$ $2.0 \mathrm{~mm}$ and $2.1-3.0 \mathrm{~mm}$, respectively. The dentin in the cusp was thinnest in the mesiobuccal and lingual pulp horns compared with the distal pulp horns (distobuccal, distolingual, and distal pulp horns) in the deciduous dentition period (Fig. 7).

\section{Discussion}

Previous studies investigating the inner morphology of teeth were performed using methods such as X-ray imaging, transparent tooth models, and superimposition of serial thin sections. However, these methods do not capture the inner features clearly and destroy the samples to a certain extent, making it difficult to reconstruct an accurate and smooth solid image.

The aim of the present study was to clarify the shape of the pulp chamber and the positional relationship between the contour of the crown and the pulp chamber of mandibular second deciduous molars using micro-CT, which allows non-invasive observation and analysis from arbitrary directions. ${ }^{16)}$ The micro$\mathrm{CT}$ technique has been widely used in recent years, especially for observation of inner structures including the shape of the pulp chamber. Whereas a number of studies have reported the morphological features of the root canal, few studies have conducted three-dimensional observations of the pulp chamber morphology. ${ }^{17-21)}$

Nozaka et al. investigated the crown and pulp chamber morphology of mandibular second deciduous molars. ${ }^{10)}$ Although thin sections were superimposed to construct a solid image for analysis, no clear stage was investigated. However, the contour of the crown and inner pulp chamber morphology is considered to change at different stages after deciduous tooth eruption and the start of occlusion. We investigated the mandibular second deciduous molars during the deciduous and mixed dentition periods to clarify the positional relationship between the crown contour and pulp chamber. The proportional volume of the pulp chamber relative to the tooth crown was calculated to objectively evaluate the morphological changes in the two dentition periods. The results showed that the proportional volume in the mixed dentition period was significantly lower than in the deciduous dentition period. Although the first molar and central incisor reached the occlusal line in the mixed dentition period, and tooth attrition of the mandibular second deciduous molar did not reach the dentin, the proportional volume of the pulp chamber was significantly lower in the mixed dentition than in the deciduous dentition period. The possible reasons considered were: 1) rapid and regular formation of secondary dentin in the entire pulp chamber with time, and 2) stimulus transmission to the dentin despite tooth attrition without reaching the dentin, causing the formation of tertiary dentin in the pulp horn. These results suggest that the formation of secondary and tertiary dentins was more active than the decrease in enamel due to tooth attrition. Changes in the dentinal thickness of each pulp horn in the deciduous and mixed dentition periods were then investigated. Since the position of the dentinoenamel junction remains unchanged, the distance between each pulp horn and the dentinoenamel junction was measured to allow comparison between the deciduous and mixed dentition periods. The results showed little difference in the mesiobuccal pulp horn between the two periods, the distance being shortest (approximately $1.5 \mathrm{~mm}$ ). In contrast, the distobuccal pulp horn showed the largest change between the two periods. Although the crown contour of the mandibular second deciduous molar was similar to that of the mandibular first molar, the occlusal surface was small due to significant narrowing of the cervical area and lingual tipping of the buccal surface. This is considered to be one of the reasons why the pulp chamber shifted to the mesial side in terms of the positional relationship between the crown contour and pulp chamber of the mandibular second deciduous molar. Since the mesiobuccal pulp horn was the highest, and the pulp chamber was elevated to the mesial side in the deciduous dentition period, extra care with regard to the mesiobuccal pulp horn is needed during cavity preparation for mandibular second deciduous molars.

In conclusion, the positional relationship between the crown contour and pulp chamber of mandibular second deciduous molars was investigated. The results obtained suggest that the mesiobuccal pulp horn projects to the largest extent and has an eminence oriented toward the buccal side, whereas the location of the pulp chamber shifts to the mesial and buccal sides relative to the crown contour. It is suggested that care is needed in order to avoid exposure of the mesiobuccal pulp horn during class 2 cavity preparation.

\section{References}

1. Ash MM and Nelson SJ. The Primary (Deciduous) Teeth. In: 9th edition Wheeler's Dental Anatomy, Physiology, and Occlusion. ed by Stanly J Nelson, Elsevier Science Philadelphia, 2010, pp 45-66.

2. Grine FE. Enamel thickness of deciduous and permanent molars in modern Homo sapiens. Am J Phys Anthropol 126: 14-31, 2005

3. Ruschel HC and Chevitarese O. A comparative study of dentin thickness of primary human molars. J Clin Pediatr Dent 27: 277-281, 2003

4. Chandler NP. The radiographic assessment of pulp size: 


\section{Yasutoyo Ikari et al.: 3D Analysis of Pulp Chamber in Deciduous Molars}

validity and clinical implications. NZ Dent J 85:23-26, 1989

5. Kandemir S. The radiographic determinability of the distance between the pulp horns in the permanent first and second molar teeth. J Oral Sci 40: 143-146, 1998

6. Lyroudia K, Pantelidou O, Mikrogeorgis G, Chatzikallinikidis C, Nikopoulos N and Pitas I. Virtual endodontics: Threedimensional tooth volume representations and their pulp cavity access. J Endodont 28: 599-602, 2002

7. Chandler NP, Pitt Ford TR and Monteith BD. Coronal pulp size in molars: a study of bitewing radiographs. Internat Endodont J 36:757-763, 2003

8. Amano M, Agematsu H, Abe S, Usami A, Matsunaga S, Suto $\mathrm{K}$ and Ide $\mathrm{Y}$. Three-dimensional analysis of pulp chambers in maxillary second deciduous molars. J Dent 34: 503-508, 2006

9. Puddhikarant P and Rapp R. Radiographic anatomy of pulpal chambers of primary molars. Pediatr Dent 5: 25-29, 1983

10. Nozaka K, Itoh M, Ono R, Satoh T, Amari E, Nozaka Y and Hongoh T. New cavity forms in second deciduous molars which maximize dentin thickness between cavity and pulp chamber. Bull Tokyo Dent Coll 31: 309-319, 1990

11. Plotino G, Grande NM, Pecci R, Bedini R, Pameijer CH and Somma F. Three-dimentional imaging using microcomputed tomography for studying tooth macromorphology. JADA 137: 1555-1561, 2006

12. Nielsen RB, Alyassin AM, Peters DD, Carnes DL and Lancaster J. An advanced system for detailed endodontic research. J Endodont 21: 561-568, 1995

13. Dowker SEP, Davis GR, Elliott JC and Wong FSL. X-ray microtomography: 3-dimensional imaging of teeth for computer-assisted learning. Eur J Dent Edu 1: 61- 65, 1997

14. Mikrogeorgis G, Lyroudia KL, Nikopolulos N, Pitas I, Molyvdas I and Lambrianidis TH. 3D computer-aided reconstruction of six teeth with morphological abnormalities. Internat Endodont J 32: 88-93, 1999

15. Sowole A, Sote E and Folayan M. Dental caries pattern and predisposing oral hygiene related factors in Nigerian preschool children. Eur Arch Paediatr Dent 8: 206-210, 2007

16. Bjorndal L, Carlsen O, Thuesen G, Darvann T and Kreiborg $\mathrm{S}$. External and internal macromorophology in 3Dreconstructed maxillary molars using computerized X-ray microtomography. Int Endodont J 32: 3-9, 1999

17. Klinge RF. A microradiographic and electron microscopic study of tertiary dentin in human deciduous teeth. Acta odont Scand 57: 87-92, 1999

18. Aboshi H, Takahashi $\mathrm{T}$ and Komuro T. Age estimation using microfocus X-ray computed tomography of lower premolars. Forensic Sci Int 200: 35-40, 2000

19. Oi T, Saka H and Ide Y. Three-dimensional observation of pulp cavities in the maxillary first premolar tooth using micro-CT. Int Endodont J 37: 46-51, 2004

20. Agematsu H, Ohnishi M, Matsunaga S, Saka H, Nakahara K and Ide Y. Three-dimensional analysis of pulp chambers in mandibular first deciduous molars. Pediat Dent J 20: 28-33, 2010

21. Changlian Du, Youjia Zhu and Li Hong. Age-related changes in pulp cavity of incisors as a determinant for forensic age identification. J Forensic Sci 56: S72-S76, 2011 
J.Hard Tissue Biology Vol. 23(2):211-216, 2014 